\title{
Evaluation of Throughput using Hybrid Cooperation
}

\author{
Meenakshi Joshi \\ Vidyalankar Polytechnic, \\ wadala 400037, University of \\ Mumbai, India.
}

\author{
J. W. Bakal,Ph.D \\ Shivajirao Jondhale college of \\ engineering, Dombivali, \\ University of Mumbai, India
}

\author{
Prof. Srikant Velankar \\ Vidyalankar Institute of \\ technology, wadala 400037 \\ University of Mumbai, India.
}

\begin{abstract}
The strength of signal becomes weak as it reaches the edge of a cell. The performance degrades due to inter-cell interference. In this paper, we present the cooperation scenario which improves this performance and calculate the signal capacity using the following schemes - without cooperation, with full and hybrid cooperation
\end{abstract}

\section{General Terms}

Base station cooperation

\section{Keywords}

Cooperative transmission, Hybrid cooperation

\section{INTRODUCTION}

During the last few decades, wireless communication systems have been under major development. The requirements have shifted from the low data rate voice services to real time video transmissions [1]. Support for higher data rates has become more essential and the development towards more advanced wireless systems is still ongoing. Multiple antennas are currently included in many of the wireless standards to achieve the required data rates. This increases the complexity of signal processing algorithms in the receiver [2]. However, the complexity and power consumption of the wireless device should be moderate. This poses challenges in developing algorithms and architectures for the mobile receiver.To achieve higher data rates channel resources must be utilized efficiently. Using frequency reuse interference can be minimized up to some limit. But, using different frequency bands for different cell decreases the spectral efficiency [9]. A new technique to provide high spectral efficiency in downlink environment is base station cooperation [2]. In a conventional cellular network, a terminal receives signals not only from the base station of that cell, but also from other cell base stations [6]. This ability to receive signals from multiple base stations can be used to improve the spectral efficiency of the cellular network and achieve higher data rates for cell edge users. In this paper, we present the cooperation scenario including 3 cases, without cooperation, with full cooperation and hybrid cooperation. Results of all 3 schemes are compared and finally decided that hybrid scheme provides better results.

There are different sections in this paper: Section 2 signal to interference noise ratio (SINR) and user throughput for cooperation and no cooperation. Section 3 describes the cooperation selection algorithm. Section 4 presents the simulation results and Section 5 presents the conclusions

\section{MODEL FOR BASE STATION COOPERATION}

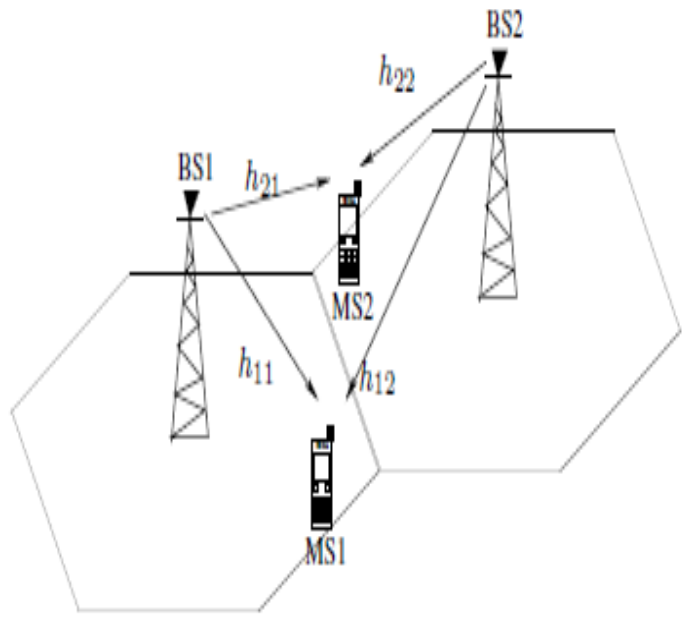

Fig. 1

The basic system model is shown in Figure 1[7]. Base stations BS1 and BS2 are in cooperation mode, which will transmit signals to mobile terminals MS1 and MS2. For base station 1 , base station 2 is one of the interfering base stations among the total 12 base stations. Here total 12 base stations are considered for simulation[3]. More than one base station can be involved in cooperation, but for simplicity we are considering only two stations to form cooperation. The signals from the serving base station and from the neighbour base station arrive at the terminal at the same time, i.e., received signal by the mobile terminal from the two base stations are frame synchronized [4]. The frame duration in which the BS1 transmits to MS1 is divided into two sub-frames, where the first sub-frame is used for signal transmission to MS1 and the second one to MS2. Similarly, BS2, which is under cooperation with BS1, transmits in the same sequence of BS1.

$\mathrm{y} 1, \mathrm{y} 2=$ received signals

hij = channel between mobile terminal $\mathrm{i}$ and base station $\mathrm{j}$.

$\mathrm{x} 1, \mathrm{x} 2=$ transmit signal of BS1 and BS2

$\mathrm{zi}=$ total interference received by MS I

$\mathrm{ni}=$ additive white Gaussian noise. 
$\left[\begin{array}{l}y 1 \\ y 2\end{array}\right]=\left[\begin{array}{ll}h 11 & h 12 \\ h 21 & h 22\end{array}\right]\left[\begin{array}{l}x 1 \\ x 2\end{array}\right]+\left[\begin{array}{l}z 1 \\ z 2\end{array}\right]+\left[\begin{array}{l}n 1 \\ n 2\end{array}\right]$

\section{A. Without Cooperation}

Under normal operation that is when there is no cooperative transmission, the signal to interference noise ratio (SINR) in the downlink for MS1 is given by

$$
\operatorname{SINR}_{\mathrm{nc}}=\frac{[h 11]^{2} \mathrm{E}\{X 1\}^{2}}{\sigma_{\mathrm{n}}^{2}+\sum_{k=2}^{12}[h 1 k] 2 E\{X 1\} 2}
$$

where $h_{i}$ represents the channel between the terminal $i$ and base station $\mathrm{j}, E \mathrm{X}_{\mathrm{i}}^{2}$ is the average transmit power of Base Station $i$, and ${ }_{n}^{2}$ is noise variance.

The capacity (or throughput) for terminal MS1 in bits/sec/Hz

$\mathrm{C}_{\mathrm{nc}}=\log 2\left(1+\mathrm{bSINR}_{\mathrm{nc}}\right)$

where, $\mathrm{b}$ is determined by the SNR gap between the practical coding scheme and the theoretical limit.

\section{B. With Cooperation}

When terminal MS1 is in cooperation with BS1 and BS2, SINR $_{\text {coop }}$, SINR of the downlink is given by

$$
\operatorname{SINR}_{\text {coop }}=\frac{(|h 11| 2+|h 12| 2) \mathrm{E}\{X 1\}^{2}}{\sigma_{\mathrm{n}}^{2}+\sum_{k=3}^{12}[h 1 k] 2 E\{X 1\} 2}
$$

The capacity (or throughput) for terminal MS1 under cooperation in bits/sec/Hz will be

$$
\mathrm{C}_{\text {oop }}=\beta \log _{2}(1+\mathrm{bSINR} \text { coop })
$$

$\beta$ defines the proportion of resource sharing among the terminals under cooperation. In our simulation, the value for $\beta$ is $1 / 2$.

\section{HYBRID COOPERATION}

User capacity under cooperation increases but not all the time.The overall (mean) capacity is increased compare to normal scheme but the individual user capacity is not increased all the time [5]. Sometimes it is worst than normal operation. To go under cooperation, it requires large backhaul signaling and extra cost in terms of complexity is also getting added [8]. Hence the question arises whether it is worth doing cooperation all the time. Therefore hybrid cooperation is introduced which provides following algorithm. This algorithm decides whether to do cooperation or simple operation.

\section{Algorithm 1 Hybrid selection}

1. Get the channel information of the serving DL and nearest DL.

2. Calculate SINR for without Cooperation scheme $\left(\mathrm{SINR}_{\mathrm{nc}}\right)$.

3. Calculate SINR for full Cooperation scheme (SINR coop$)$.

4. Case: Low SINR regime

5. For $\operatorname{SINR}_{\mathrm{nc}}<=0$ Do

6. If $\operatorname{SINR}_{\text {coop }}>2 * \mathrm{SINR}_{\mathrm{nc}}$ then

7. Base station selects Cooperation mode.

8. Else

9. Base station selects normal operation mode.

10. End if

11. End for

12. Case: High SINR regime

13. For $\operatorname{SINR}_{n c}>=0$ do

14. If $\operatorname{SINR}_{\text {coop }}>\operatorname{SINR}_{\mathrm{nc}}{ }^{2}$ then

15. Base station selects Cooperation mode.

16. Else

17. Base station selects normal operation mode.

18. End if

19. End for

\section{SIMULATION AND RESULTS}

Tab1e 1

\begin{tabular}{|l|l|}
\hline Scheme & $\begin{array}{l}\text { Mean } \\
\text { Capacity } \\
\text { (bits/Sec/Hz) }\end{array}$ \\
\hline Full Cooperation & 0.3508 \\
\hline Hybrid Cooperation & 0.5532 \\
\hline High region & 0.4583 \\
\hline
\end{tabular}


In hybrid cooperation, full cooperation is selected only when SINRnc is smaller than SINRcoop as given in algorithm. In this simulation we have considered 12 base stations with one user per cell edge. The average power transmitted by serving base station as $10 \mathrm{~dB}$, noise variance as $5 \mathrm{~dB}$ and average interfering power of base stations is $6 \mathrm{~dB}$, according to path loss model COST 231 is considered [6]. The mean throughput or capacity and mean SINR for cell edge user's under No cooperation, Full cooperation and hybrid cooperation scheme is shown in Table 1. Throughput captured from first 1000 frames is shown in Fig. 2, Which describes Hybrid cooperation with red colour gives better result as compared to other 2 schemes.

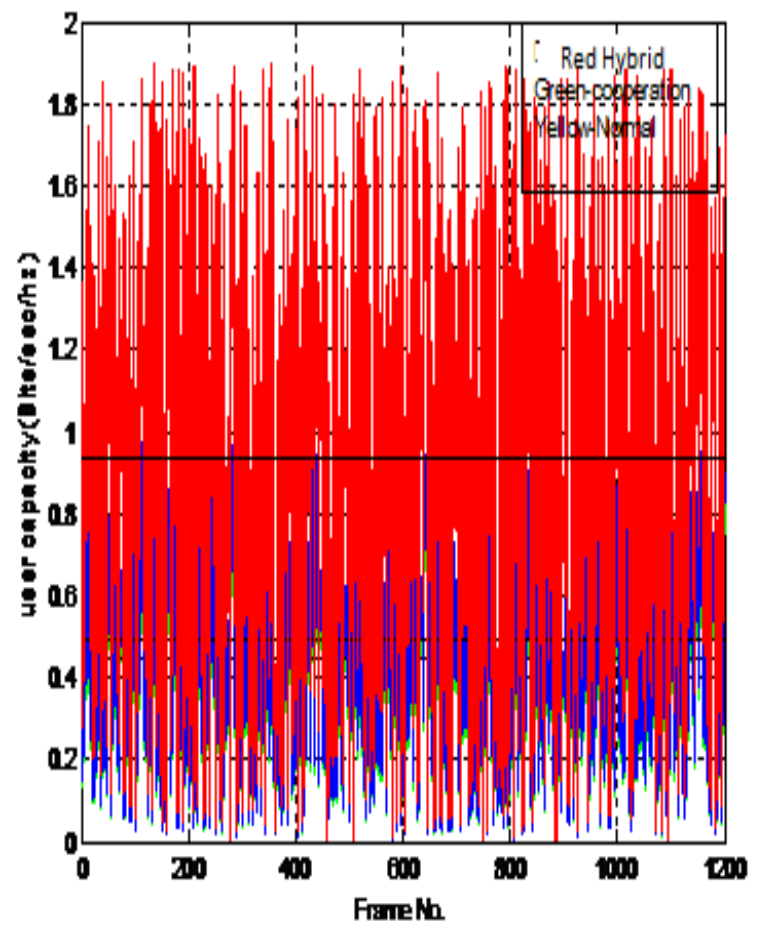

Fig. 2 Comparison of all 3 schemes

\section{CONCLUSIONS}

In this paper, simulation analysis of hybrid cooperation in a cellular network was presented. The simulation results show that under normal operation the cell edge user SINR and Capacity is quite poor due to interference. In full cooperation although the interference is removed, the user capacity of a cell-edge is not always better than normal transmission due to bad channel condition. By using hybrid method, both capacity and SINR is improved. The improvement is about $61.70 \%$ from full cooperation, which was $33 \%$ in previous research. According to the result, the proposed hybrid scheme provides a better result compared to full-time cooperation.

\section{REFERENCES}

[1] Sari, H.; Sezginer, S.; "MIMO techniques and full frequency reuse in mobile WiMAX systems," Telecommunications, 2009. ICT 09. International Conference on, vol., no., pp.8-12,2527May2009

[2] Boudreau, G.; Panicker, J.; Ning Guo; Rui Chang; Neng Wang; Vrzic, S.; , "Interference coordination and cancellation for 4G networks," Communications Magazine, IEEE , vol.47, no.4, pp.74-81, April 2009

[3] J. G. Andrews, W. Choi and R. W. Heath, "Overcoming Interference in Spatial Multiplexing MIMO Cellular Networks", IEEE WirelessCommunications Magazine, vol. 14, no. 6 , pp.95-104, Dec 2007

[4] T.Tamaki, K. Seong and J. M. Cioffi,"Downlink MIMO Systems Using Cooperation among Base Stations in a Slow Fading Channel", Proceeding of IEEE International Conf. on Communications 2007, pp. 4728-4733, June 2007.

[5] Ramesh Kumar, M.R. Bhashyam, S. Jalihal, D.,"Throughput improvement for cell-edge users using selective cooperation in cellular networks," 5th IFIP International Conference on Wireless and Optical Communications Networks, 2008. WOCN '08, vol, no.pp.1-5, 5-7 May 2008 doi: 10.1109/WOCN.2008.4542516

[6] Tolli, A.Pennanen, H. Komulainen, P , "On the Value of Coherent and Coordinated Multi-Cell Transmission," IEEE International Conference on Communications Workshops, ICC Workshops 2009, vol., no., pp.1-5, 14-18 June 2009

[7] Yong SooCho, Jaekwon Kim Book on "MIMO-OFDM wireless communications with MATLAB"ISBN 978$0-470-82561-7$

[8] Jeffrey G. Andrews,Book on "Fundamentals of WiMAX Understanding Broadband Wireless Networking'ISBN 0-13-222552-2

[9] Engstrom, S.; Johansson, T.; Kronestedt, F.; Larsson, M.; Lidbrink, S.; Olofsson, H.; "Multiple reuse patterns for frequency planning in GSM networks," Vehicular Technology Conference, VTC 98. 48th IEEE, vol.3, no., pp.2004-2008 vol.3, 18-21 May 1998 